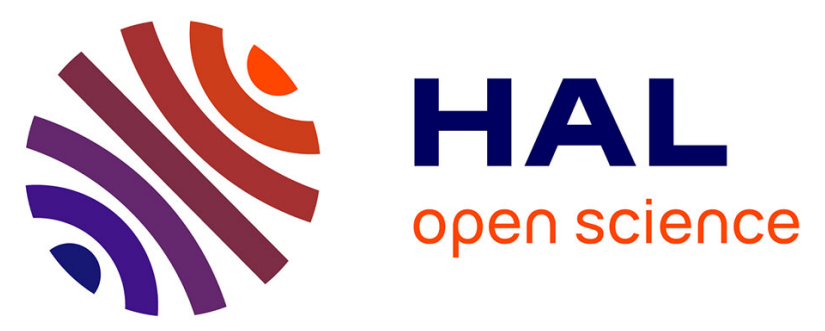

\title{
Assemblages bois-fer et biocorrosion: étude des sulfures de fer formés en conditions anoxiques dans des bois d'épaves
}

Céline Remazeilles, Francois Leveque, Egle Conforto, Philippe Refait

\section{- To cite this version:}

Céline Remazeilles, Francois Leveque, Egle Conforto, Philippe Refait. Assemblages bois-fer et biocorrosion: étude des sulfures de fer formés en conditions anoxiques dans des bois d'épaves. Matériaux \& Techniques, 2017, 104 (5), 10.1051/mattech/2017006 . hal-02345846

HAL Id: hal-02345846

https://hal-univ-rochelle.archives-ouvertes.fr/hal-02345846

Submitted on 4 Nov 2019

HAL is a multi-disciplinary open access archive for the deposit and dissemination of scientific research documents, whether they are published or not. The documents may come from teaching and research institutions in France or abroad, or from public or private research centers.
L'archive ouverte pluridisciplinaire HAL, est destinée au dépôt et à la diffusion de documents scientifiques de niveau recherche, publiés ou non, émanant des établissements d'enseignement et de recherche français ou étrangers, des laboratoires publics ou privés. 


\section{Assemblages bois-fer et biocorrosion : étude des sulfures de fer formés en conditions anoxiques dans des bois d'épaves}

Céline Rémazeilles $^{\mathrm{a}^{*}}$, François Lévêque ${ }^{\mathrm{b}}$, Egle Conforto $^{\mathrm{a}}$, Philippe Refait ${ }^{\mathrm{a}}$

${ }^{a}$ LaSIE, Laboratoire des Sciences de l'Ingénieur et de l'Environnement, UMR 7356 CNRS/Université de La Rochelle

bLIENSs, Littoral, Environnement et Sociétés, UMR 7266 CNRS/Université de La Rochelle

\section{Résumé}

Les conséquences de la corrosion des renforts de fer dans les assemblages de bois peuvent compromettre la durabilité d'une structure. Si le bois est gorgé d'eau, les conditions à la surface du métal deviennent anoxiques. La présence de fer et l'activité microbiologique favorisée par la présence de matière organique font des systèmes composites bois-fer des milieux propices à l'apparition de sulfures de fer. Or ces derniers se transforment soit en milieux anaérobies sulfurés, soit lorsque l'oxygène diffuse dans le bois, soit lors d'une remise à l'air de la structure, avec pour conséquence de contribuer à la dégradation du bois. Afin de mieux comprendre les mécanismes impliqués, nous étudions des bois d'épaves archéologiques. Cet article sera illustré par des exemples d'époques récentes $\left(18^{\mathrm{è}}\right.$ s. $19^{\grave{e}}$ s.) et antiques et par une approche analytique multi-techniques originale. La mackinawite, la greigite et la pyrite ont été identifiées et il semble que la nature des sulfures de fer présents soit liée à l'âge du vestige conformément aux schémas proposés dans la littérature d'évolution de ces composés. La greigite est 
intéressante à considérer. Seule phase détectée quel que soit l'âge, elle peut s'avérer un bon marqueur de la biocorrosion du fer dans les assemblages bois-fer.

\section{Mots clés :}

\section{Fer/bois/biocorrosion/sulfures de fer/greigite/ propriétés magnétiques}

\section{Introduction:}

La pérennité des assemblages bois-fer sur le long terme est problématique dès lors qu'ils sont gorgés d'eau. Si le problème est bien connu des restaurateurs et conservateurs du patrimoine, il semble qu'il soit encore peu abordé dans le domaine du génie civil contemporain.

Ainsi les bois archéologiques gorgés d'eau sont soumis à de nombreux phénomènes de dégradation post-fouille de natures :

(i) physique : le séchage entraîne la précipitation de sels capables d'occasionner des contraintes mécaniques suffisamment importantes pour fissurer le bois et occasionne aussi l'apparition d'efflorescences inesthétiques en surface [1].

(ii) chimique : les macromolécules du bois, notamment la cellulose et l'hémicellulose sont très sensibles à l'hydrolyse acide et à l'oxydation. Cette dernière peut éventuellement être catalysée par le fer [2-4]. 
(iii) biologique : en tant que matière organique, le bois fournit des nutriments, voire alimente directement de nombreux micro/macro-organismes vivants. En plus de cela, lorsque des pièces de bois assemblées par des renforts de fer séjournent en milieux anoxiques et biologiquement actifs, ils subissent des dommages dramatiques une fois exposés à l'air. Pendant l'enfouissement, les bactéries sulfurogènes réduisent les composés oxygénés de soufre (sulfates, sulfites, etc.), présents dans l'environnement, en espèce sulfurées $\left(\mathrm{H}_{2} \mathrm{~S}, \mathrm{HS}^{\top}\right)$ [5]. Celles-ci, combinées avec les ions fer(II) résultant de la corrosion des objets en fer utilisés pour l'assemblage, peuvent précipiter en sulfures de fer(II). La chimie des sulfures de fer(II) est extrêmement complexe et implique des transformations de phases aussi bien anoxiques (durant l'enfouissement) que contrôlées par l'oxygène (diffusion de l'oxygène dans le système sur site ou après mise au jour) [6]. Les composés fer/soufre s'avèrent influencer fortement les mécanismes de dégradation du bois comme constaté dans de nombreux vestiges archéologiques, notamment des épaves. Les dommages post-fouilles des bois archéologiques associés à la formation de sulfures de fer consistent principalement en la précipitation de cristaux volumineux de composés fer/soufre (ex : mélantérite $\left[\mathrm{FeSO}_{4}, 7 \mathrm{H}_{2} \mathrm{O}\right]$, jarosite $\left.\left[\mathrm{KFe}_{3}(\mathrm{OH})_{6}\left(\mathrm{SO}_{4}\right)_{2}\right]\right)$ et en une forte acidité particulièrement néfaste à la résistance du bois qui finit par perdre ses propriétés mécaniques. Le cas le plus emblématique de cette problématique est celui du Vasa, navire suédois du $17^{\text {è }}$ siècle [7-8]. Ayant sombré dans le port de Stockholm le jour de son voyage inaugural en 1628, il a été renfloué en 1961 puis a été traité au polyéthylène glycol pendant 17 ans (+ 9 ans de séchage). Le polyéthylène 
glycol, couramment utilisé pour la conservation des vestiges archéologiques en bois gorgés d'eau, a pour fonction de maintenir la structure en préservant l'intégrité mécanique du matériau organique [1]. Venant en remplacement de l'eau, il ne s'agit pas d'un traitement chimique ; son action est essentiellement physique. Malgré cette campagne de traitement de vaste ampleur, le Vasa est à l'heure actuelle en train de s'affaisser sur lui-même dans le musée qui lui est dédié, du fait d'une contamination générale par les composés fer/soufre et d'une extrême acidité du bois. D’autres épaves historiques sont concernées de la même façon comme le Mary Rose (16 ${ }^{\text {ème }}$ siècle) ou le Batavia (17 ${ }^{\text {ème }}$ siècle) [9-12]. La pyrite $\left(\mathrm{FeS}_{2}\right)$ et le soufre minéral $\left(\alpha-\mathrm{S}_{8}\right)$ sont les principaux composés fer/soufre mentionnés dans la littérature sur les bois archéologiques humides [7-12]. Mais la mackinawite $(\mathrm{FeS})$ et la greigite $\left(\mathrm{Fe}_{3} \mathrm{~S}_{4}\right)$ ont également été détectées dans des teneurs significatives lors d'une étude que nous avons menée il y a quelques années dans le cadre du programme de recherche PrévArch (2008-2010, financement du Ministère de la Culture et de la Communication) en partenariat avec les laboratoires Arc-Nucléart et Arc'Antique [13].

Le présent article reprend quelques résultats du programme PrévArch afin de les confronter à une étude plus récente menée sur une épave du $4^{\text {ème }}$ siècle. Cette dernière a été découverte dans ce qui était à l'époque antique l'ensemble portuaire de Narbonne dans le sud de la France. Le site est maintenant à l'intérieur des terres et le sol est gorgé d'eau. Une campagne de prospection magnétique réalisée sur le site en 2014 a révélé que le bois était anormalement magnétique. Il a également été déterminé qu'il ne reste plus de métal dans les clous utilisés pour l'assemblage. 
Par conséquent, le signal magnétique a été supposé provenir de produits de corrosion magnétiques ayant diffusé à l'intérieur du bois. Plusieurs composés contenant du fer comme la magnétite $\left(\mathrm{Fe}_{3} \mathrm{O}_{4}\right)$, la maghémite $\left(\gamma-\mathrm{Fe}_{2} \mathrm{O}_{3}\right)$, la greigite $\left(\mathrm{Fe}_{3} \mathrm{~S}_{4}\right)$, la pyrrhotite $\left(\mathrm{Fe}_{(1-\mathrm{x})} \mathrm{S}\right)$ et la smithyte $\left(\mathrm{Fe}_{9} \mathrm{~S}_{11}\right)$ sont naturellement magnétiques. Une étude a donc été réalisée afin d'identifier les phases minérales présentes dans le bois, et d'être en mesure d'attribuer le signal magnétique à une ou plusieurs d'entre elles.

Cet article présente les résultats analytiques obtenus par une combinaison de méthodes d'imagerie électronique, d'analyses structurales et élémentaires. Mais l'originalité de cette démarche est d'utiliser, en outre des méthodes de caractérisation magnétique pour la détection de certains sulfures de fer. La nature des sulfures de fer obtenus dans les différents vestiges étudiés sera discutée et confrontée aux schémas d'évolution de la mackinawite, considérée comme le premier précipité en cas de biocorrosion. Un autre enjeu est d'évaluer la pertinence de l'utilisation des méthodes magnétiques pour du diagnostic non destructif.

\section{Matériaux et méthodes:}

\section{Corpus :}

Les travaux présentés dans cet article portent sur un ensemble d'épaves suspectées d'avoir été contaminées par le fer et le soufre.

L'USS Monitor est un cuirassé américain du $19^{\text {ème }}$ siècle qui a servi durant la guerre de sécession. Fait de bois (chêne) et d'acier, il a sombré en 1862. Une 
partie gît encore au large de la côte Est des Etats Unis, une autre est conservée en eau au Mariner's Museum (Newport News, Virginie). Les conservateurs du Mariner's Museum nous ont fait parvenir des fragments de ce qui est gardé au musée.

La Natière, dans la baie de St Malo, rassemble deux épaves : l'Aimable Grenot et La Dauphine, datant du $18^{\text {ème }}$ siècle. Elles ont été fouillées par le DRASSM pendant dix ans (1999-2008) et de nombreux objets ont été remontés. Parmi les restes sélectionnés pour une restauration, des fragments de bois ont été sacrifiés pour notre étude. Nous ne savons pas quel fragment provient de l'une ou de l'autre épave. Nous mentionnerons ces échantillons comme ayant été extraits des « épaves de La Natière ».

L'épave dite de Mandirac, se trouve dans un site correspondant aux ports antiques de Narbonne (site de Castelou/Mandirac). Ce site terrestre a été découvert en 1945 grâce à des photographies aériennes. En 2007, une étude géophysique a confirmé la présence des restes enfouis d'infrastructures d'un système portuaire fluviolagunaire. Un programme de fouilles lancé en 2010 a mis au jour les restes d'un bateau avec son chargement d'amphores enchâssé dans le quai [14,15]. En fait, le bateau a été délibérément coulé au cours du $4^{\text {ème }}$ siècle, afin de consolider le quai. Plusieurs indicateurs laissaient, là aussi, supposer la présence de sulfures de fer comme le sol gorgé d'eau, le signal magnétique émis par le bois. Des concrétions de pyrites étaient même visibles à l'emplacement de restes de clous.

Méthodologie analytique: 
Les échantillons extraits de l'USS Monitor et des épaves de La Natière ont été étudiés lors du projet PrévArc (2008-2010). L'épave de Mandirac a été étudiée plus tard (2015). La méthodologie analytique a donc évolué entre temps, en ce sens que pour l'étude de l'épave de Mandirac des méthodes de caractérisation magnétique ont été introduites. Nous regrouperons donc sous l'appellation de «méthodes classiques» la micro-spectroscopie Raman ( $\mu$-SR), la microscopie électronique à balayage couplée à la spectroscopie en dispersion d'énergie (MEB/EDS) et la diffraction des rayons X (DRX), comparées aux «méthodes magnétiques », à notre connaissance jamais utilisées dans un tel contexte.

Dans un premier temps, des lamelles fraîchement coupées de l'échantillon d'origine ont été examinées avec un stéréomicroscope (Leica, M165C) pour des observations macroscopiques et pour localiser des zones intéressantes en vue de la micro-analyse.

Les analyses en micro-spectroscopie Raman ont été réalisées avec un spectromètre Jobin Yvon Haute Résolution (HR LabRAM) équipé d'un microscope (Olympus BX41) et d'un objectif x50. La puissance du laser a été atténuée pour éviter la transformation par chauffage des phases minérales thermosensibles et les spectres ont été enregistrés à une résolution de $0,2 \mathrm{~cm}^{-1}$. Les échantillons ont été étudiés avec une longueur d'onde d'excitation de 632,82 nm.

Les micrographies électroniques haute résolution et les compositions élémentaires ont été obtenues avec un microscope SEM-FEI, Quanta 200 FEG/ESEM couplé avec un système EDS EDAX Genesis pour la microanalyse X. Les observations 
ont été effectuées en mode environnemental, à basse pression $(0,002 \mathrm{~atm})$ et avec une tension d'accélération pouvant varier entre 15 et $20 \mathrm{kV}$. Ce mode expérimental ne nécessite pas de préparation pour les échantillons nonconducteurs et les échantillons de bois ont été placés encore humides dans la chambre d'analyse.

Les analyses en diffraction des rayons $\mathrm{X}(\mathrm{DRX})$ ont été réalisées directement sur le bois. Pour cela une lamelle fraîchement coupée a été placée dans le porteéchantillon et exposée au faisceau de rayons X. Selon les épaves, deux diffractomètres différents ont été utilisés. Les bois de 1'USS Monitor et des épaves de La Natière ont été analysés avec un diffractomètre Bruker AXS ${ }^{\circledR}$ D8 Advanced avec une longueur d'onde $\lambda_{\mathrm{Cu}-\mathrm{K} \alpha}=0,15406 \mathrm{~nm}$. La vaigre 11 de l'épave de Mandirac a été étudié avec un diffractomètre INEL EQUINOX 6000 avec une longueur d'onde $\lambda_{\mathrm{Co}-\mathrm{K} \alpha}=0,17903 \mathrm{~nm}$.

Enfin, des mesures de $\mathrm{pH}$ ont été effectuées pour chaque échantillon sur une tranche fraîchement coupée encore humide (électrode de surface Sentix Sur WTW).

En outre, pour l'épave de Mandirac, des mesures de susceptibilité magnétique en champ faible ont été effectuées sur de cubes de $8 \mathrm{~cm}^{3}$ avec un susceptibilimètre KLY4, Agico. Puis des courbes d'aimantation rémanente isotherme (IRM) ont été acquises : une première acquisition directe puis une seconde en sens opposé (dite backfield) jusqu'à 3T atteignant la saturation de l'aimantation (générateur de champ à impulsions magnétiques MMPM10 Magnetic Measurements). L'aimantation résultante à chaque pallier d'aimantation a été mesurée avec un 
magnétomètre JR6 Agico. La susceptibilité magnétique présente une large gamme de variation sur trois ordres de grandeur, des valeurs négatives à positives fortes. Une constante arbitraire de $25.10^{-6}$ SI est ajoutée afin de procéder à une transformée en $\log _{10}$. Cela permet de ne considérer que les composants para- et ferro-magnétiques s.l. en supprimant les composés diamagnétiques, à savoir le bois et l'eau.

\section{$\underline{\text { Sulfures de fer attendus : }}$}

Le tableau 1 regroupe les sulfures de fer présents dans la nature avec l'état d'oxydation du fer et du soufre et leurs propriétés magnétiques respectives. La magnétite et la maghémite, qui ne sont pas des sulfures, ont été ajoutées à cette liste du fait de leur caractère magnétique. Notons que dans un contexte de corrosion de métaux ferreux archéologiques, seules la mackinawite, la greigite et la pyrite ont été mentionnées dans la littérature [16-18]. Toutefois les autres phases ne peuvent être exclues. Les méthodes magnétiques sont extrêmement sensibles. Elles peuvent détecter des espèces magnétiques à des teneurs inférieures au ppm et peuvent donc déceler la présence d'espèces non mises en évidence jusque-là par les méthodes classiques, qui n'atteignent pas une telle précision.

\section{Échantillonnage:}

Les échantillons de l'USS Monitor et des épaves de La Natière consistaient entre autres, en des fragments de bois gorgés d'eau, de taille variant entre 5 et $10 \mathrm{~cm}$ de 
long, de formes non définies, soit découpés d'un élément plus grand de l'épave soit récupérés tels quels. Il était très important de conserver ces fragments humides. Ils nous ont donc été transmis emballés dans du film plastique ou dans des sachets placés dans des boîtes hermétiques remplies d'eau du réseau public de distribution. Après réception, nous les avons conservés dans leur conditionnement d'origine, au réfrigérateur à $4^{\circ} \mathrm{C}$ durant toute la campagne d'analyses. Les expériences ( $\mu$-SR et $\mathrm{DRX})$ ont été réalisées sans protection particulière contre l'air ambiant, sur des petites lamelles découpées au scalpel dont seule la facette interne était présentée au faisceau incident. Cette découpe était réalisée juste avant l'analyse et renouvelée pour chaque méthode (également en MEB/EDS) de sorte que soit toujours étudiée une surface non exposée à l'air et fraichement découpée. Cette précaution visait à éviter une oxydation des sulfures de fer par l'oxygène de l'air.

Concernant l'épave de Mandirac, plusieurs planches gorgées d'eau ont été extraites. Afin de préserver les composés sensibles à l'oxygène, les planches ont été enveloppées dans un film plastique après extraction et congelés à $-4^{\circ} \mathrm{C}$ au laboratoire jusqu'à l'analyse. Les résultats présentés dans cet article ont été obtenus sur la planche appelée vaigre 11 . Cette planche était d'environ $80 \mathrm{~cm}$ de long, $16,5 \mathrm{~cm}$ de large et $2,5 \mathrm{~cm}$ d'épaisseur. Afin de convenir d'un échantillonnage optimisé, une cartographie de susceptibilité magnétique de la planche a été préalablement réalisée, en lignes et en colonnes (susceptibilimètre portable SM-30, ZH instruments) (Fig. 1). Une zone de forte intensité du signal magnétique matérialisée en rouge apparaît sur cette cartographie. La couleur bleue 
superposée au reste de la planche ne signifie pas que le bois ne présente pas de signal ferromagnétique mais plutôt qu'il l'est beaucoup moins que la zone rouge. En effet toute la planche montre des propriétés ferromagnétiques s.l.. Ensuite, la planche a été entièrement découpée en cubes de $2 \times 2 \times 2 \mathrm{~cm}^{3}$ avec une scie circulaire munie d'une lame à flasque téflon. Tous les cubes ont été analysés avec les méthodes magnétiques mais seuls treize ont été complètement analysés (méthodes magnétiques + méthodes classiques). Ceux-ci ont été choisis le long de la ligne e et de la colonne 20 dans le prolongement du point le plus magnétique en position e20. Les cubes ont été nommés en fonction de leur position lignecolonne.

\section{Résultats}

Résultats significatifs du programme PrévArc (2008-2010):

Les échantillons de l'USS Monitor et des épaves de La Natière ayant été analysés dans le cadre du programme PrévArc, les résultats ont été déjà publiés [13,18]. Nous reprendrons les plus significatifs pour cet article, à savoir la nature des sulfures de fer mis en évidence à l'intérieur des fragments de bois. La Figure 2 regroupe les micrographies MEB, les spectres EDS, les spectres $\mu$-SR et les diffractogrammes obtenus pour deux fragments différents de l'USS Monitor (S0178 et S-0179) et deux fragments différents des épaves de La Natière (2006-189 et 2006-072). Les micrographies de la Figure 2, notamment celles concernant les échantillons S-0178 et S-0179, montrent que le bois contient de nombreuses concrétions cristallines. Celles-ci se distinguent en clair sur les micrographies qui 
sont représentées en contraste chimique. La taille des cristaux est de l'ordre de quelques micromètres, voire moins. Les concrétions sont regroupées dans les canaux conducteurs de la sève, le bois apparaissant en contraste plus foncé, du fait des éléments légers $(\mathrm{C}, \mathrm{O})$ qui constituent la matière organique. Sur la base des analyses EDS, ces concrétions sont riches en soufre et en fer. Les spectres Raman montrent essentiellement la mackinawite. Cette phase, de formule $\mathrm{Fe}^{\mathrm{II}} \mathrm{S}$, a la particularité de posséder trois spectres Raman selon son état cristallin et son état d'oxydation. Les spectres de la Figure 2 montrent que ces trois spectres caractéristiques ont été obtenus à partir des échantillons analysés, voire se superposent sur le spectre global. L'association des pics à $208 \mathrm{~cm}^{-1}$ et $282 \mathrm{~cm}^{-1}$ est caractéristique de la mackinawite à l'état nanocristallin. L'association des pics à $208 \mathrm{~cm}^{-1}$ et $298 \mathrm{~cm}^{-1}$ est liée à de la mackinawite dite «cristalline » [19,20]. Enfin les pics à $125,175,256,312,322 \mathrm{~cm}^{-1}$ et l'épaulement à $355 \mathrm{~cm}^{-1}$ sont attribués à une forme partiellement oxydée de la mackinawite dans laquelle une faible proportion du fer est ferrique [20-21]. La greigite, caractérisée par l'ensemble de bandes à 188, 250, 350, $365 \mathrm{~cm}^{-1}$ a été régulièrement mise en évidence [21]. Il est toutefois difficile d'affirmer si la greigite détectée était initialement présente dans les échantillons ou si elle résulte d'une oxydation de la mackinawite qui se serait produite durant l'analyse ou le transport. Enfin, des pics ont été attribués au soufre minéral (indexés $\alpha-S_{8}$ sur la Figure 2), qui peut également résulter de l'oxydation de la mackinawite [20]. Les diffractogrammes de la Figure 2 sont en accord avec les spectres Raman. Ils montrent clairement les pics de la mackinawite qui semble être le composé prédominant. La greigite est également détectée. Des bandes 
larges apparaissent sur les diffractogrammes à $16,2^{\circ}$ et $22,5^{\circ}$. Elles correspondent au bois.

Il est à noter que la pyrite n'a été détectée par aucune méthode utilisée. Alors que cette phase était attendue car souvent mentionnée dans la littérature, elle s'avère absente des échantillons de l'USS Monitor et de ceux de La Natière.

\section{L'épave de Mandirac:}

Les résultats de l'identification des composés minéraux pour la vaigre 11 de l'épave de Mandirac, obtenus pour les 13 cubes analysés complètement, sont résumés dans le Tableau 2. A titre d'exemples représentatifs, seuls les résultats des cubes e4, e14, e20 et h20 sont détaillés dans cet article. La Figure 1 montre les courbes d'aimantation rémanente isotherme directe et backfield des cubes localisés sur la cartographie de susceptibilité magnétique. Ces courbes sont très similaires et se superposent les unes aux autres, signifiant que les propriétés magnétiques des cubes sont données par les mêmes espèces. En fait ces courbes sont caractéristiques de la greigite et permettent en outre de déterminer des granulométries d'environ 20 à 500 nm [22]. La teneur en greigite, a été extrapolée

à partir de la valeur théorique d'aimantation rémanente à saturation (Ms $=59$ $\mathrm{Am}^{2} \mathrm{~kg}^{-1} ; \mathrm{Mrs} / \mathrm{Ms}=0,12 ;$ [23]) et varie de 0,3 à $6000 \mathrm{ppm}$ en fonction de la distance avec le cube présentant le signal le plus intense (e20). Ainsi, la greigite est la seule espèce magnétique détectée par les méthodes magnétiques. La magnétite et la maghémite semblent absentes. La Figure 3 regroupe les images obtenues sous stéréomicroscope, les micrographies MEB, les spectres Raman et 
les diffractogrammes des cubes e4, e14, e20 et h20. Sur les images en lumière naturelle, des particules noires apparaissent dans les fibres de bois des échantillons e4 et e14. Des liserés dorés sont également observés le long des fibres de bois des échantillons e20 et h20, suggérant la présence de pyrite. La micrographie MEB de l'échantillon e4 montre des petits cristaux clairs disséminés dans le bois. Les spectres Raman et le diffractogramme ont permis de déterminer qu'il s'agit d'un mélange de grains de pyrite et de marcassite. Au fur et à mesure que l'on se rapproche de la zone la plus magnétique, les phases minérales commencent à former des agglomérats de grains micrométriques à submicrométriques (échantillon e14). Une forte densité de framboïdes est ensuite observée dans la zone la plus magnétique (cubes e20, h20). Les analyses EDS ont confirmé que les cristaux clairs observés en contraste chimique étaient principalement composés de soufre et de fer avec une moyenne du rapport atomique $\mathrm{Fe} / \mathrm{S}$ égal à $0,51 \pm 0,04$. Cette valeur est en adéquation avec la stœchiométrie $\mathrm{FeS}_{2}$ de la pyrite et de la marcassite. Les morphologies de grains sont variées. La Figure 4 montre des exemples de morphologies et de répartition des cristaux dans les fibres de bois. La marcassite se reconnaît à sa structure de croissance radiale (Fig. 4(a)). La pyrite montre, quant à elle, toute une gamme de morphologies, cubes, cubes tronqués (Fig. 4(b)), octaédres, octaèdres tronqués, dodécaèdres, framboïdes (Fig. 4(c)et 4(d)). Or, la morphologie des nanocristaux de pyrite pourrait dépendre de la teneur en soufre et/ou en fer du milieu [24]. Quoiqu'il en soit, les grains et framboïdes se retrouvent confinés dans les canaux conducteurs de la sève et s'agglomèrent pour les remplir en prenant la forme de la 
paroi (Fig. 4(e)et 4(f)). Les spectres Raman et les diffractogrammes confirment la présence de pyrite (bandes Raman à 344, 380 et $430 \mathrm{~cm}^{-1}$ ) et de marcassite (bandes Raman à 323 et $386 \mathrm{~cm}^{-1}$ ). La marcassite a été principalement détectée dans les cubes e4 et e5 et la pyrite était prédominante dans tous les autres. La greigite a été largement détectée en spectroscopie Raman dans les échantillons e20, f20, g20 et h20. Elle a même été mise en évidence par diffraction des rayons X. Mais les méthodes classiques ne l'ont mise en évidence que dans la zone la plus magnétique alors que les méthodes magnétiques l'ont détectée dans toute la planche. De la sidérite $\left(\mathrm{FeCO}_{3}\right)$ a été identifiée dans les échantillons g20 et h20 (bandes Raman à 185, 283, 715 et $1085 \mathrm{~cm}^{-1}$ ) (Fig.3). En fait, l'échantillon g20 contient les restes d'un clou complètement corrodé et entièrement transformé en un mélange hétérogène de pyrite et de sidérite. La sidérite détectée dans le bois provient donc du reste de ce clou. Par ailleurs des cristaux aciculaires composés de soufre, d'oxygène et de calcium ont parfois été observés. Il s'agit de gypse $\left(\mathrm{CaSO}_{4} \cdot 2 \mathrm{H}_{2} \mathrm{O}\right)$ qui a été identifié par $\mu$-SR et DRX. Ces cristaux résultent certainement du séchage des échantillons durant l'analyse. L'évaporation de l'eau concentre les sels qui précipitent souvent en gypse dans ce type de systèmes [713]. Enfin, une ligne de base importante apparaît sur les diffractogrammes ainsi que de larges bandes à $18,7^{\circ}$ et $26,7^{\circ}$ ou $32,6^{\circ}$. Ce signal, comme précédemment, est attribué au bois (remarque : les angles de diffraction diffèrent de ceux observé pour l'USS Monitor et les épaves de La Natière car une longueur d'onde différente a été utilisée). 
Enfin, les mesures de pH sont rassemblées sur la Figure 5 pour les échantillons de l'ensemble des épaves. On constate que pour les échantillons de l'USS Monitor et des épaves de La Natière, le liquide d'imprégnation du bois présentait des valeurs comprises entre 7,0 et 4,7. Concernant l'épave de Mandirac, les résultats montrent que le bois humide est légèrement acide au niveau de l'échantillon e4, avec un $\mathrm{pH}$ de 5,6. Il devient de plus en plus acide au fur et à mesure que l'on s'approche de la zone la plus magnétique pour laquelle le pH est aux alentours de 3. La valeur de $\mathrm{pH}$ la plus faible $(2,6)$ correspond au cube g20 contenant le reste de clou.

\section{Discussion}

Les sulfures de fer dans les assemblages bois/fer archéologiques gorgés d'eau :

Les échantillons de l'USS Monitor et des épaves de la Natière contiennent principalement de la mackinawite. La présence de greigite pour ces épaves est à considérer avec prudence du fait de la possible oxydation de la mackinawite durant les analyses. Concernant la vaigre 11 de l'épave de Mandirac, la greigite a été révélée par les méthodes de caractérisation magnétique dans les zones où les méthodes classiques n'ont pas permis de la détecter. Les mesures magnétiques ont d'ailleurs démontré que le signal magnétique en rémanence de la vaigre 11 était seulement dû à la greigite. Cela peut être extrapolé à l'ensemble de l'épave. Il reste que le composé prédominant dans le bois de l'épave de Mandirac est la pyrite. Les grains montrent toute une gamme de morphologies mais on note une forte densité de pyrite framboïdale autour du reste de clou. Wilkin et Barnes (1997) expliquent 
la formation de la pyrite framboïdale à partir de la précipitation de la mackinawite qui se transforme ensuite en greigite. Les microcristaux de greigite s'aggloméreraient du fait de leurs propriétés magnétiques pour se transformer en pyrite une fois la framboïde formée. Celle-ci peut continuer à croître pour donner des grains plus gros et des agglomérats moins ordonnés [25]. Les sulfures de fer sont donc présents en forte densité autour du reste de clou comme l'ont montré les micrographies MEB pour la pyrite et les mesures magnétiques pour la greigite. Leur répartition suppose également une diffusion dans le bois via les canaux des tissus vasculaires. Ainsi en se répandant dans le bois depuis tous les clous de l'épave, les sulfures de fer ont fini pas l'envahir entièrement. La présence de marcassite, conjointement à la pyrite, est difficile à expliquer. Nous ne pouvons préciser si elle fait partie intégrante du schéma global d'évolution de la mackinawite, comme décrit dans la Figure 6, ou si elle s'est formée de manière indépendante à ce processus. Enfin les signaux magnétiques de la pyrrhotite, de la troilite ou de la smythite n'ont pas été détectés. Les méthodes magnétiques étant extrêmement sensibles, nous considérons qu'ils ne sont pas présents dans la vaigre 11 ou alors à l'état de trace par rapport à la greigite.

\section{$\underline{\text { La nature des sulfures de fer renvoie à l'âge du vestige: }}$}

On constate donc que la nature des sulfures de fer peut varier selon les épaves. Une explication peut venir d'une différence majeure que l'on peut constater dans notre corpus : l'âge du vestige. L'USS Monitor et les épaves de La Natière sont 
relativement récentes $\left(19^{\text {ème }}\right.$ et $18^{\text {ème }}$ siècles $)$ comparées à l'épave de Mandirac (4 ${ }^{\text {ème }}$ siècle). Or, le schéma d'évolution des sulfures de fer en conditions anoxiques est tout à fait compatible avec le facteur temps. La Figure 6 résume les schémas d'évolution de la mackinawite en conditions anoxiques ou en cas d'exposition à l'air. La chimie des sulfures de fer a été largement étudiée. Leur présence dans un contexte de corrosion du fer est principalement expliquée par une activité microbiologique. La mackinawite est considérée comme le premier précipité en cas de biocorrosion du fer influencée par les bactéries anaérobies sulfurogènes. Par la suite, les paramètres tels que le caractère oxique/anoxique de l'environnement, l'approvisionnement en soufre via la production d'espèces sulfurées, la température, l'humidité, le $\mathrm{pH}$, etc. déterminent une voie ou une autre [20, 21, 25-30]. Sans apport en sulfures et en milieu anoxique, la mackinawite peut être considérée comme stable. En cas d'apport constant en sulfures, accompagné d'une baisse du $\mathrm{pH}$, la mackinawite ( 1 atome de fer pour 1 atome de soufre) est transformée en greigite ( 1 atome de fer pour 1,3 atomes de soufre) puis en pyrite ( 1 atome de fer pour 2 atomes de soufre). Ce schéma est parfaitement compatible avec le scénario d'une activité microbiologique prolongée et ce quelle que soit la nature du site d'enfouissement. Les bactéries sulfurogènes se développent aussi bien en milieu marin que dans un sol, dès lors que le milieu est saturé en eau et contient suffisamment de sulfates [31]. Dans les assemblages bois/fer, la présence abondante de matière organique, le milieu faiblement oxygéné voire complètement anoxique et l'eau constituent un terrain particulièrement fertile pour le développement des colonies de bactéries 
anaérobies sulfurogènes. Avec en plus un objet en fer en train de se corroder, tous les facteurs d'un puissant réacteur pour la production de sulfures de fer sont réunis. De plus, la transformation de la mackinawite en pyrite via la greigite assure, étape par étape, une assimilation progressive du soufre par les phases solides, de sorte que le milieu aqueux n'atteint jamais un niveau de surdose en sulfures qui pourrait inhiber le développement de colonies de bactéries sulfurogènes. Ainsi l'activité microbiologique peut se maintenir pendant très longtemps et la présence abondante de pyrite et de greigite dans l'épave de Mandirac semble corroborer cette hypothèse.

\section{$\underline{\text { La greigite et le magnétisme pour diagnostiquer la biocorrosion : }}$}

La limite de détection des méthodes de caractérisation magnétique est très faible (inférieure au ppm) et celles-ci sont en mesure d'analyser des échantillons dans la masse, tandis que les méthodes classiques reposent sur l'analyse de surface. Ainsi, les méthodes magnétiques complètent les méthodes classiques dans une approche analytique exhaustive visant à identifier des phases minérales à l'intérieur d'une matrice organique.

Par ailleurs, la greigite est une phase particulièrement intéressante à considérer. D’après le schéma de la Figure 6, elle peut se produire de manière intermédiaire (i) dans le processus de transformation de la mackinawite en pyrite en environnement anoxique et (ii) lors de l'oxydation à l'air de la mackinawite. Elle est donc susceptible d'être présente dans de nombreuses circonstances. Il s'agit en effet du seul sulfure de fer détecté dans tous les échantillons que nous avons 
analysés, quel que soit l'âge du vestige. On peut alors considérer la greigite comme un marqueur de la biocorrosion dans les assemblages bois/fer gorgés d'eau. En outre, de par ses propriétés magnétiques, il est possible de la détecter facilement. La caractérisation magnétique est donc envisageable comme méthode non destructive pour diagnostiquer la biocorrosion via la détection de la greigite, dans les assemblages bois/fer gorgés d'eau, et pas seulement archéologiques. En effet, la prise de conscience actuelle de l'impact des activités humaines sur l'environnement amène nos sociétés à privilégier des ressources renouvelables comme le bois. Or les constructions bois réalisées notamment en milieu littoral, lacustre ou fluvial ne sont pas à l'abri de phénomènes de biocorrosion dès lors que les pièces sont assemblées par des renforts d'acier. On peut alors en toute logique penser que cela se manifesterait par la formation de mackinawite, phase instable dont l'évolution est associée à une baisse du $\mathrm{pH}$. Or le bois est fragilisé en conditions acides. La question du risque potentiel que constituerait la biocorrosion sur l'ensemble d'une structure moderne fabriquée à partir d'un assemblage bois/fer se pose donc. A notre connaissance cela n'a encore jamais été évalué.

\section{Conclusion}

Des assemblages bois/fer archéologiques humides ont été analysés. Il s'agit de bois d'épaves assemblés par des clous en fer et ayant séjourné soit en eau de mer soit dans un sol gorgé d'eau. Ce type de systèmes est un terrain propice à la biocorrosion. Or les conséquences pour le bois peuvent être désastreuses, 
notamment du fait de la baisse importante du pH qui accompagne le phénomène et qui a pour conséquence de détruire la cellulose et l'hémicellulose. Les sulfures de fer ont été identifiés et leur nature semble dépendre de l'âge du vestige. Les épaves du $18^{\text {ème }}$ et $19^{\text {ème }}$ siècle contiennent de la mackinawite et de la greigite. L'épave antique ( $4^{\text {ème }}$ siècle) contient de la greigite et de la pyrite. La présence de ces composés est conforme aux étapes d'évolution de la mackinawite, premier précipité à apparaitre lors de la biocorrosion des métaux ferreux. Celle-ci a pu se former lors de la biocorrosion des clous d'assemblage puis se transformer dans un environnement anoxique constamment enrichi en soufre par une activité prolongée des bactéries sulfurogènes. Par ailleurs la greigite est le seul sulfure de fer détecté quel que soit l'âge de l'épave. Cette phase peut donc être considérée comme un marqueur de la biocorrosion. Elle peut cependant être présente en trop faible quantité pour être mise en évidence à l'aide de méthodes d'analyse «classiques» comme la spectroscopie Raman, la microscopie électronique à balayage et la diffraction des rayons X. Notre étude démontre que les méthodes de caractérisation magnétique sont bien plus efficaces car la greigite est ferromagnétique $s . l$. Les méthodes de caractérisation magnétique sont en effet extrêmement sensibles et ont permis de détecter la greigite à des teneurs inférieures au ppm. Elles pourraient alors être utilisées comme techniques d'analyse non destructives pour diagnostiquer la biocorrosion dans des assemblages bois/fer humides, via la détection de la greigite. 


\section{Remerciements}

Nous remercions chaleureusement Corinne Sanchez (Archéologie des Sociétés Méditerranéennes, UMR-5140 CNRS/Université de Montpellier 3/Ministère de la Culture et de la Communication/Labex Archimède), Marie-Pierre Jézégou (DRASSM), Eric Nordgren et Susan Grieve (The Mariners' Museum) qui nous ont permis d'obtenir des échantillons archéologiques sacrifiables pour notre étude. Le projet PrévArch (2008-2010) a été financé par le Ministère de la Culture et de la Communication, piloté par Arc-Nucléart (Khôi Tran) en partenariat avec Arc'Antique (Elodie Guilminot et Sylvie Labroche).

Nous remercions enfin Maylis Minjacq (étudiante) et Guillaume Lotte (LaSIE) pour leur contribution à l'étude des échantillons de l'épave de Mandirac. 


\section{Références}

[1] Y. Fors, F. Jalilehvand, M. Sandström, Analytical Aspects of Waterlogged Wood in Historical Shipwrecks, Analytical Sciences 27 (2011) 785-792.

[2] E. Farber, Chemical Deterioration of Wood in the Presence of Iron, Industrial and Engineering Chemistry 46 (1954) 1968-1972.

[3] A.J. Baker, Degradation of wood by products of metal corrosion, USDA Forest Service research paper FPL 229 (1974) 1-6.

[4] J.A. Emery, H.A. Schroeder, Iron-Catalyzed Oxidation of Wood Carbohydrates, Wood Science and Technology 8 (1974) 123-137.

[5] J. Le Gall, Bactéries sulfato-reductrices: enzymologie de la réduction dissimilative des sulfates, Plants and Soil 43 (1975) 115-124.

[6] R. Walker, Instability of iron sulfides on recently excavated artifacts, Studies in Conservation 46 (2001) 141-152.

[7] M. Sandström, F. Jalilehvand, I. Persson, U. Gelius, P. Frank, I. Hall-Roth, Deterioration of the Seventeenth-century Warship Vasa by Internal Formation of Sulphuric Acid, Nature 415 (2002) 893-897.

[8] Y. Fors, M. Sandström, Sulfur and iron in shipwrecks cause conservation concerns, Chemical Society Reviews 35 (2006) 399-415.

[9] M. Sandström, F. Jalilehvand, E. Damian, Y. Fors, U. Gelius, M. Jones, M. Salomé, Sulfur Accumulation in the Timbers of King Henry VIII's Warship Mary Rose: A Pathway in the Sulfur Cycle of Conservation Concern, In: H.B. Gray, ed. 
National Academy of Science of the United States of America, Proceedings. Pasadena: California Institute of Technology 102 (2005) 14165-14170.

[10] K.M. Wetherall, R.M. Moss, A.M. Jones, A.D. Smith, T. Skinner, D.M.

Pickup, S.W. Goatham, A.V. Chadwick, R.J. Newport, Sulfur and Iron Speciation in Recently Recovered Timbers of the Mary Rose revealed via X-Ray Absorption Spectroscopy, Journal of Archaeological Science 35 (2008) 1317-1328.

[11] I.D. Mac Leod, C. Kenna, Degradation of Archaeological Timbers by Pyrite: Oxidation of Iron and Sulphur Species. In: P. Hoffmann, ed. ICOM Committee for Conservation Working Group on Wet Organic Archaeological Materials, 4th ICOM Group on Wet Organic Archaeological Materials Conference, Proceedings. Bemerhaven, (1991) 133-41.

[12] I. Godfrey, V. Richards, I. MacLeod, The Batavia - Past, Current and future conservation. In: M. Ek, ed. Chemistry and preservation of waterlogged wooden shipwrecks, Shipwrecks 2011, Proceedings. Stockholm: Royal Institute of technology, (2011) $22-28$.

[13] C. Rémazeilles, K. Tran, E. Guilminot, E. Conforto, P. Refait, Study of Fe(II) sulphides in waterlogged archaeological wood, Studies in Conservation 58 (2013) 297-307.

[14] M.P. Jézégou, J. Cavéro, M. Druez, H. Günter-Martin, V. Mathé, C. Sanchez, K. Storch, A geo-archaeological research about the Roman Harbours of Narbonne: earth and underwater survey and GIS. Ikuwa 3: Beyond Boundaries, 3rd International Congress on Underwater Archaeology, London, 2008, Dr. Rudolf Habelt GmbH (2012) 299-307. 
[15] C. Sanchez, C. Faïsse, M.P. Jézégou, V. Mathé, Le système portuaire de Narbonne antique : approche géoarchéologique, Dans L. Mercuri, González R. Villaescusa, F. Bertoncello (dir.), Implantations humaines en milieu littoral Méditerranéen : facteurs d'installation et processus d'appropriation de l'espace, de la Préhistoire au Moyen Âge, 24e rencontres internationales d'archéologie et d'histoire d'Antibes, 2013, éditions APDCA (2014) 125-136.

[16] V. Fell, J. Williams, Iron sulphides Corrosion products on artifacts from waterlogged deposits, Proceedings of the Interim Meeting of the ICOM-CC Metal WG, Draguignan (1998) 111-115

[17] C. Remazeilles, A. Dheilly, S. Sable, I. Lanneluc, D. Neff, P. Refait, Microbiologically influenced corrosion process of archaeological iron nails from the sixteenth century, Corrosion Engineering, Science and Technology 45 (2010) 388-394

[18] C. Rémazeilles, M. Saheb, D. Neff, E. Guilminot, K. Tran, J.A. Bourdoiseau, R. Sabot, M. Jeannin, H. Matthiesen, P. Dillmann, P. Refait, Microbiologically influenced corrosion of archaeological artefacts: characterization of iron(II) sulfides by Raman spectroscopy, Journal of Raman Spectroscopy 41 (2010) 11351143.

[19] M. Mullet, S. Boursiquot, M. Abdelmoula, J.M. Génin, J.J. Ehrhard, Surface chemistry and structural properties of mackinawite prepared by reaction of sulfide ions with metallic iron, Geochimica Cosmochimica Acta 66 (2002) 829-836. 
[20] J.A. Bourdoiseau, M. Jeannin, R. Sabot, C. Rémazeilles, Ph. Refait, Characterisation of mackinawite by Raman spectroscopy: Effects of crystallisation, drying and oxidation, Corrosion Science 50 (2008) 3247-3255.

[21] J.A. Bourdoiseau, M. Jeannin, C. Rémazeilles, R. Sabot, Ph. Refait, The transformation of mackinawite into greigite studied by Raman spectroscopy, Journal of Raman Spectroscopy 42 (2011) 496-504.

[22] A.P. Roberts, L. Chang, C. J. Rowan, C.S. Horng F. Florindo.. Magnetic properties of sedimentary greigite $\left(\mathrm{Fe}_{3} \mathrm{~S}_{4}\right)$ : an update, Reviews of Geophysics 49 (2011) $1-46$

[23] L. Chang, A.P. Roberts, Y. Tang, B.D. Rainford, A.R. Muxworthy, Q. Chen, Fundamental magnetic parameters from pure synthetic greigite $\left(\mathrm{Fe}_{3} \mathrm{~S}_{4}\right)$. Journal of Geophysical Research 113 (2008) 1-16.

[24] A.A. Barnard, S.P. Russo, Modelling nanoscale $\mathrm{FeS}_{2}$ formation in sulfur rich conditions, Journal of Materials Chemistry 19 (2009) 3389-3394

[25] R.T. Wilkin, H.L. Barnes, Formation processes of framboidal pyrite, Geochimica et Geocosmochimica Acta 66 (1997) 323-339

[26] S. Hunger, L. G. Benning, Greigite: a true intermediate on the polysulfide pathway to pyrite, Geochemical Transactions 8 (2007) 1-20.

[27] D. Rickard, G.W. Luther, Kinetics of pyrite formation by the $\mathrm{H}_{2} \mathrm{~S}$ oxidation of iron (II) monosulfide in aqueous solutions between 25 and $125^{\circ} \mathrm{C}$ : The mechanism. Geochimica et Cosmochimica Acta 61 (1997) 135-147.

[28] R.M. Garrels, M.E. Thompson, Oxydation of pyrite by iron sulfate solutions American Journal of Science 258 (1960) 57-67 
[29] W. Stumm, J.J. Morgan, Aquatic chemistry: an introduction emphasizing chemical equilibria in natural waters, New York, Wiley-Interscience, 1981 [30] P.J. Sullivan, K.J. Reddy, J.L. Yelton, Iron sulfide oxidation and the chemistry of acid generation. Environmental Geological Water Science 11 (1988) 289-295.

[31] C. Chatelus, Contribution à l'étude du rôle des bactéries sulfato-réductrices dans les phénomènes de biocorrosion, Thèse, Université de Technologie de Compiègne, 1987. 


\section{Figures}

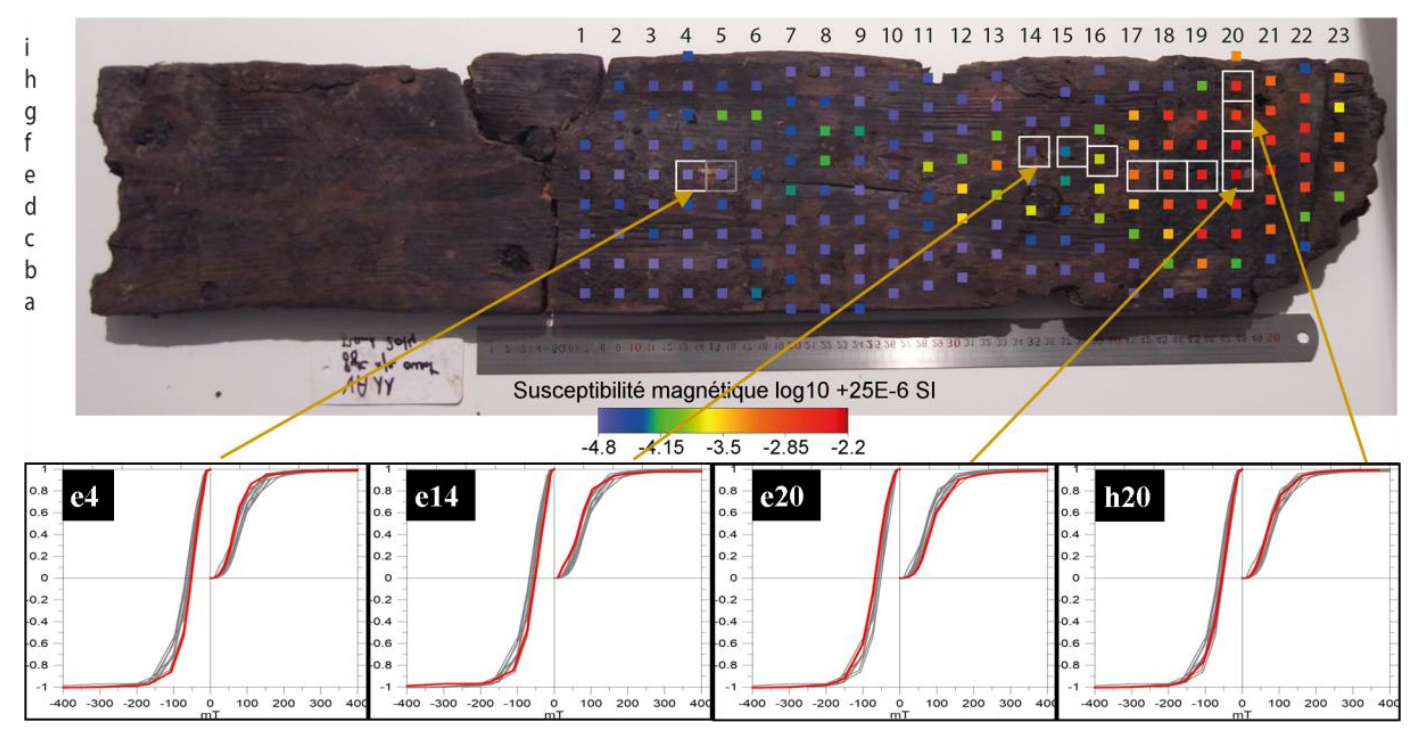

Figure 1 : Cartographie de la susceptibilité magnétique de la vaigre 11 extraite de l'épave de Mandirac. Courbes d'aimantation permanente (aimantation rémanente isotherme) des cubes échantillons e4, e14, e20 et h20. 

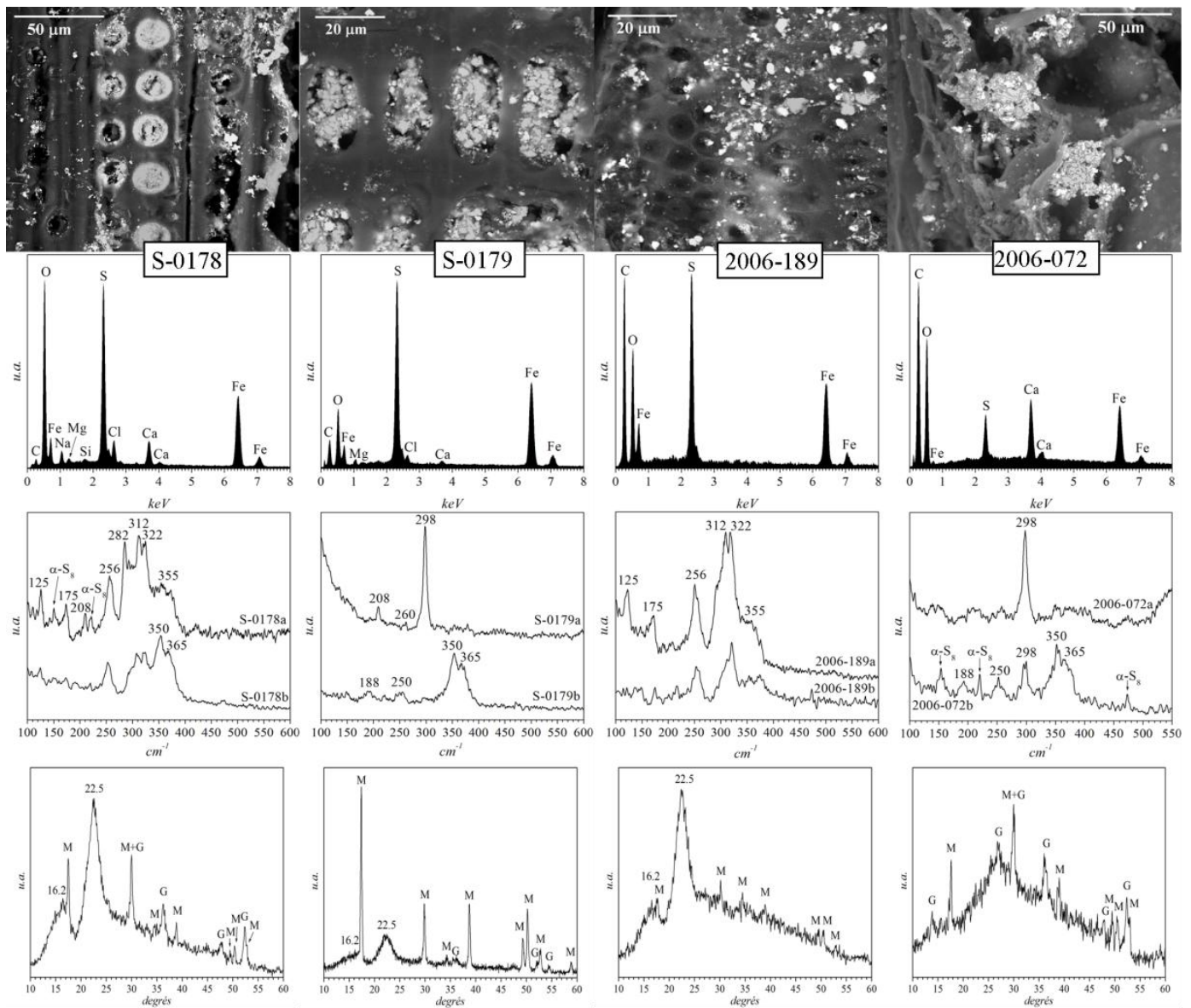

Figure 2: En lignes du haut vers le bas: micrographies MEB (contraste chimique), spectres EDS, spectres Raman, diffractogrammes d'échantillons étudiés dans le cadre du Programme PrévArch (2008-2010). En colonnes de gauche à droite: échantillons S-0178 (USS Monitor), S-0179 (USS Monitor), 2006-189 (La Natière), 2006-072(La Natière) [13]. $\alpha-S_{8}=$ soufre minéral, $M=$ mackinawite, $\mathrm{G}=$ greigite. 

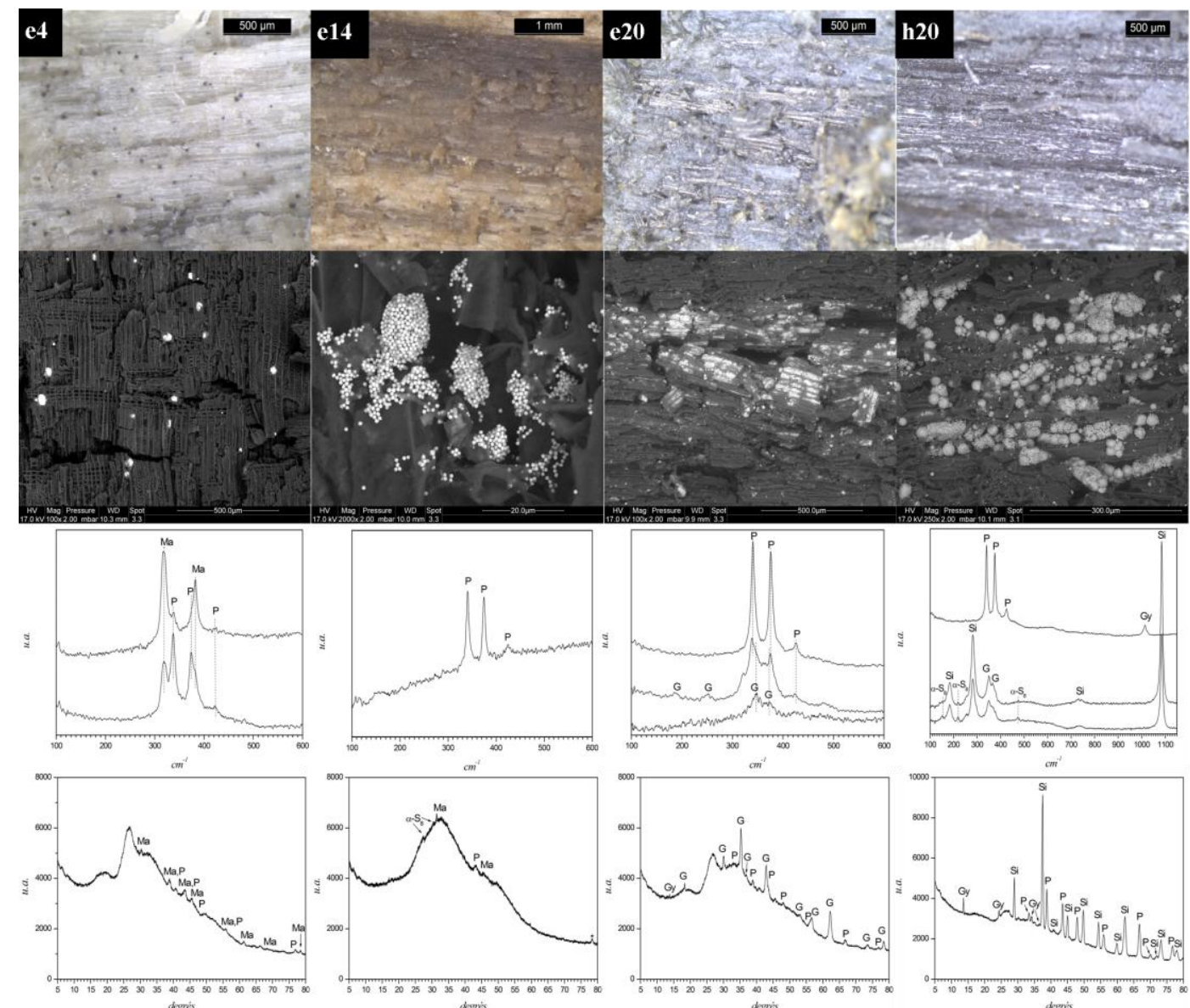

Figure 3 : En lignes du haut vers le bas : photographies sous stéréomicroscope, micrographies MEB (contraste chimique), spectres Raman, diffractogrammes. En colonnes de gauche à droite : cubes échantillons e4, e14, e20, h20 de l'épave de Mandirac. $\mathrm{Ma}=$ marcassite, $\mathrm{P}=$ pyrite, $\mathrm{G}=$ greigite, $\mathrm{Gy}=$ gypse, $\mathrm{Si}=$ siderite . 


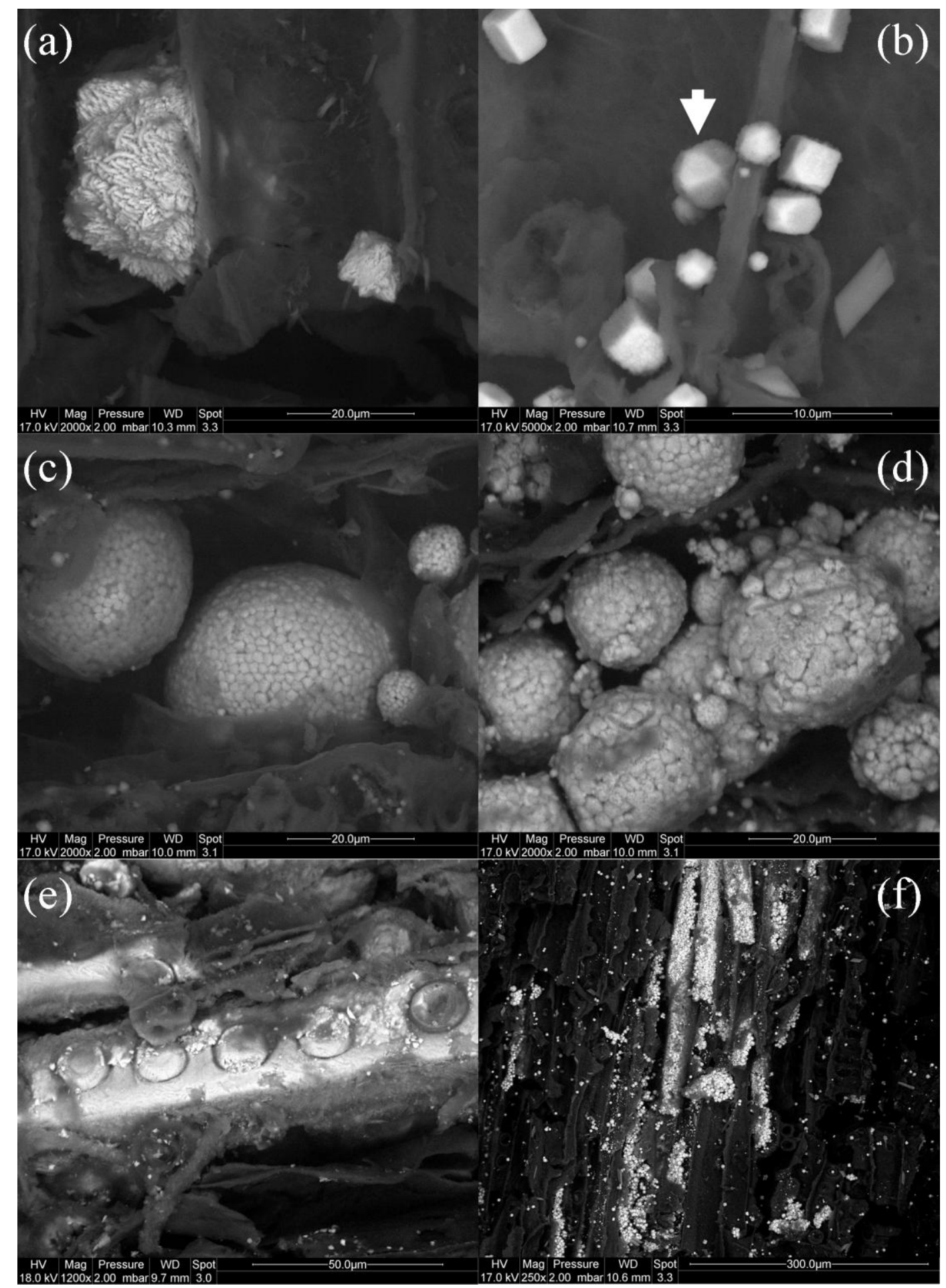

Figure 4 : Exemples de morphologies et répartition de la pyrite dans le bois de l'épave de Mandirac observées au MEB en contraste chimique. (a) grain de marcassite, (b) grains de pyrite montrant une morphologie cubique et cubique tronquée (flèche), (c) pyrite framboïdale, (e) et (f) pyrite remplissant les vaisseaux du bois. 


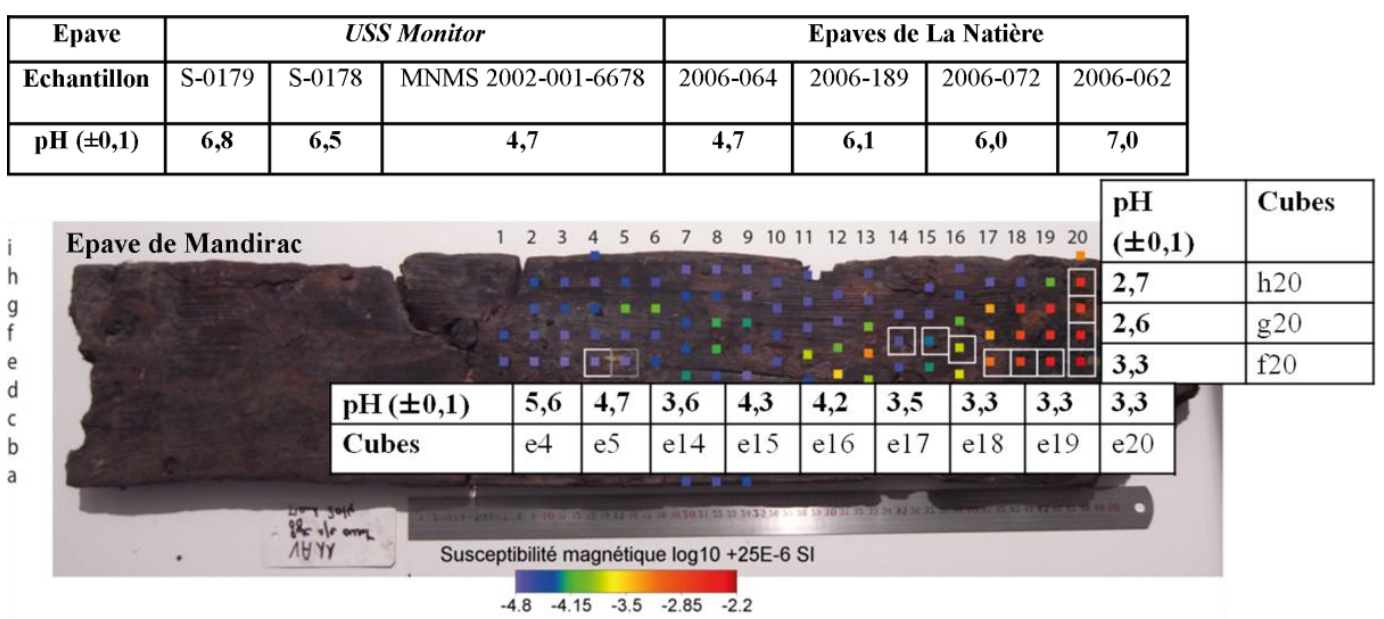

Figure 5 : Mesures de $\mathrm{pH}$ des échantillons de l'USS Monitor, des épaves de La Natière et des cubes échantillons de l'épave de Mandirac.

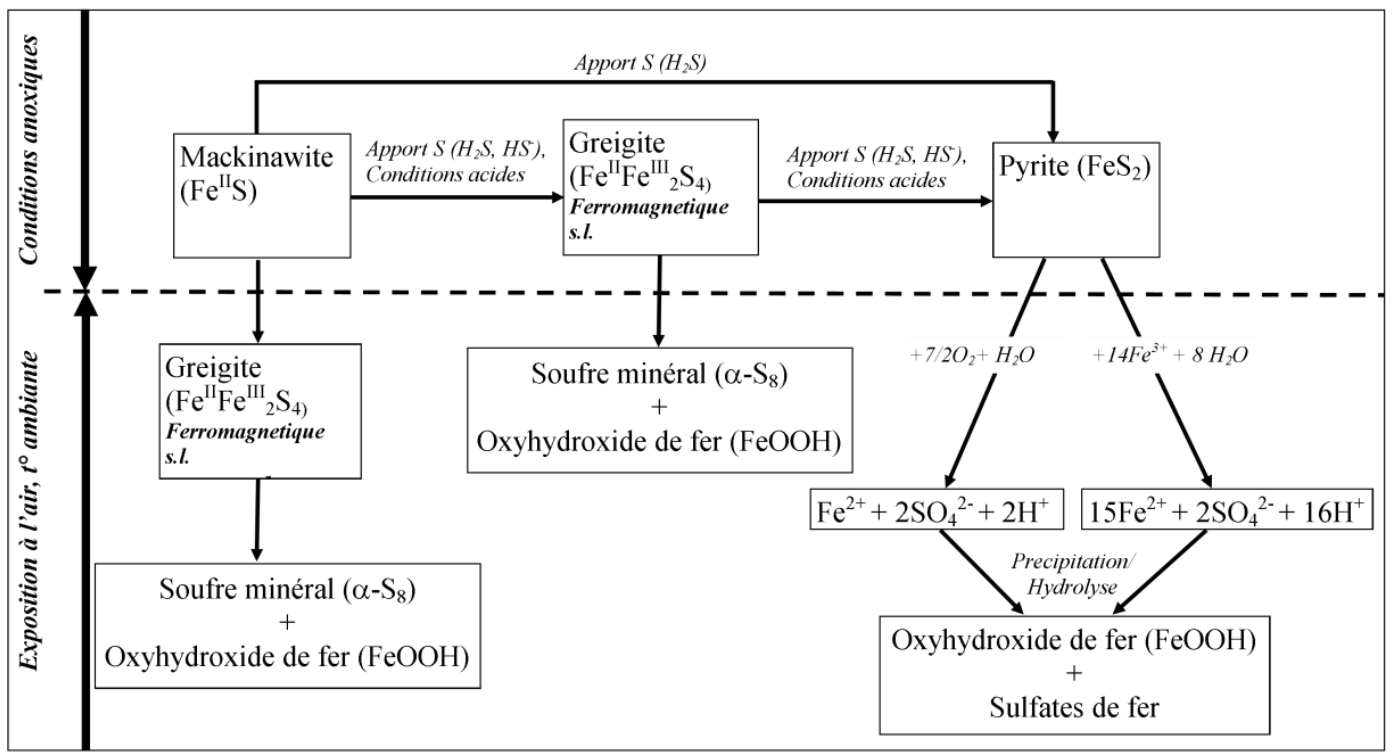

Figure 6. Schémas d'évolution et d'oxydation de la mackinawite en conditions anoxiques [20, 21, 25, 26, 27] et exposée à l'air ambiant [20, 21, 26, 28, 29, 30]. 


\section{Tableaux}

Tableau 1 : Sulfures de fer présents dans la nature, formule, état d'oxydation et propriétés magnétiques (la magnétite et la maghémite ont été ajoutées du fait de leur caractère magnétique)

\begin{tabular}{|l|l|l|l|}
\hline Minéral & Formule & Nombres d'oxydation & Propriétés magnétiques \\
\hline Mackinawite & $\mathrm{FeS}$ & $\mathrm{Fe}(+\mathrm{II}), \mathrm{S}(-\mathrm{II})$ & Paramagnétique \\
\hline Troilite & $\mathrm{FeS}$ & $\mathrm{Fe}(+\mathrm{II}), \mathrm{S}(-\mathrm{II})$ & Paramagnétique \\
\hline Pyrrhotite & $\mathrm{Fe}_{1-\mathrm{x}} \mathrm{S}$ & $\mathrm{Fe}(+\mathrm{II}), \mathrm{S}(-\mathrm{II})$ & Ferromagnétique s.l. \\
\hline Greigite & $\mathrm{Fe}_{3} \mathrm{~S}_{4}$ & $\mathrm{Fe}(+\mathrm{II},+\mathrm{III}), \mathrm{S}(-\mathrm{II})$ & Ferromagnétique s.l. \\
\hline Smythite & $\mathrm{Fe}_{9} \mathrm{~S}_{11}$ & $\mathrm{Fe}(+\mathrm{II},+\mathrm{III}), \mathrm{S}(-\mathrm{II})(?)$ & Ferromagnétique s.l. \\
\hline Pyrite & $\mathrm{FeS}_{2}$ & $\mathrm{Fe}(+\mathrm{II}), \mathrm{S}(-\mathrm{I})$ & Paramagnétique \\
\hline Marcassite & $\mathrm{FeS}_{2}$ & $\mathrm{Fe}(+\mathrm{II}), \mathrm{S}(-\mathrm{I})$ & Paramagnétique \\
\hline Magnétite & $\mathrm{Fe}_{3} \mathrm{O}_{4}$ & $\mathrm{Fe}(+\mathrm{II},+\mathrm{III}), \mathrm{O}(-\mathrm{II})$ & Ferromagnétique s.l. \\
\hline Maghémite & $\mathrm{Fe}_{2} \mathrm{O}_{3}$ & $\mathrm{Fe}(+\mathrm{III}), \mathrm{O}(-\mathrm{II})$ & Ferromagnétique s.l. \\
\hline
\end{tabular}

Tableau 2. Composition minérale des cubes échantillons de l'épave de Mandirac complètement étudiés

\begin{tabular}{|c|c|c|}
\hline $\begin{array}{l}\text { Cubes } \\
\text { échantillons }\end{array}$ & $\begin{array}{l}\text { Phases minérales détectées } \\
\text { par les méthodes classiques }\end{array}$ & $\begin{array}{l}\text { Phases minérales détectées par } \\
\text { les méthodes magnétiques }\end{array}$ \\
\hline $\mathrm{e} 4$ & Pyrite, Marcassite & Greigite \\
\hline e5 & Pyrite, Marcassite & Greigite \\
\hline e14 & Pyrite, Gypse & Greigite \\
\hline e15 & Pyrite, Soufre $\left(\alpha-S_{8}\right)$ & Greigite \\
\hline e16 & $\begin{array}{l}\text { Pyrite, Marcassite, Gypse, } \\
\text { Soufre }\left(\alpha-S_{8}\right)\end{array}$ & Greigite \\
\hline e17 & Pyrite, Soufre $\left(\alpha-S_{8}\right)$, Greigite & Greigite \\
\hline e18 & Pyrite, Soufre $\left(\alpha-S_{8}\right)$, Greigite & Greigite \\
\hline e19 & Pyrite, Greigite, Gypse & Greigite \\
\hline $\mathrm{e} 20$ & $\begin{array}{l}\text { Pyrite, Greigite, Gypse, } \\
\text { Soufre }\left(\alpha-S_{8}\right)\end{array}$ & Greigite \\
\hline $\mathrm{f} 20$ & Pyrite, Soufre $\left(\alpha-S_{8}\right)$, Greigite & Greigite \\
\hline g20 & $\begin{array}{l}\begin{array}{l}\text { Pyrite, } \\
\text { Greigite, }\end{array} \quad \text { Soufre } \quad\left(\mathrm{FeCO}_{3}\right) \text {, }\left(\alpha-\mathrm{S}_{8}\right) \text {, } \\
\text { Rozénite }\left(\mathrm{FeSO}_{4} \cdot 4 \mathrm{H}_{2} \mathrm{O}\right)\end{array}$ & Greigite \\
\hline $\mathrm{h} 20$ & $\begin{array}{l}\text { Pyrite, Sidérite, Greigite, } \\
\text { Soufre }\left(\alpha-S_{8}\right) \text {, Gypse }\end{array}$ & Greigite \\
\hline $\mathrm{i} 20$ & Pyrite, Gypse & Greigite \\
\hline
\end{tabular}

